\title{
SANS MEASUREMENT OF DEUTERIUM TRAPPING AT DISLOCATIONS AND GRAIN BOUNDARIES IN PALLADIUM
}

\author{
B. J. HEUSER ${ }^{1} \dagger$, J. S. KING ${ }^{1}$, G. C. SUMMERFIELD ${ }^{1}$, F. BOUE ${ }^{2}$ and J. E. EPPERSON ${ }^{3}$ \\ 'Department of Nuclear Engineering, University of Michigan, Ann Arbor, MI 48109, U.S.A., \\ ${ }^{2}$ Laboratoire Léon Brillouin CEN-Saclay, France and ${ }^{3}$ Materials Science Division, Argonne National \\ Laboratory, Argonne, IL 60439, U.S.A.
}

(Received 31 July 1990; in revised form 12 February 1991)

\begin{abstract}
Abotract--Small angle neutron scattering (SANS) measurements have been made on deformed single crystal and polycrystalline palladium with and without deuterium dissolved in the solution phase at room temperature. The net scattering cross section for the same sample with and without deuterium loading shows a behavior expected from deuterium correlation with dislocations forming rod-like scattering structures. We conclude from these scattering measurements that the trapped deuterium is within $2-3$ Burgers vectors of the dislocation core. On average 1-3 deuterons per $\AA$ are trapped at dislocations in the deformed samples. The measurements indicate the straight, rod-like correlation geometry extends on average $50-100 \AA$ along the dislocations. Net scattering from a well annealed polycrystalline palladium sample exhibiting a behavior expected from surfaces has been observed. This net scattering is attributed to deuterium trapped at grain boundaries in the polycrystalline sample. A value of $0.4 \pm 0.2$ trapped deuterons per $\AA^{2}$ of grain boundary area is calculated from the well annealed polycrystalline scattering measurement.
\end{abstract}

Remume-On effectue des mesures de diffusion neutronique aux petits angles sur un monocristal de palladium et sur du palladium polycristallin déformés avec et sans deutérium dissous dans la phase en solution à la température ambiante. La section efficace de diffusion pour le même échantillon avoc et sans addition de deutérium révèle un comportement attendu d'aprés la corrélation qui existe entre le deutérium et les dislocations qui forment des structures diffusantes en baguette. On conclut, à partir de ces mesures de diffusion, que le deutérium piégé se trouve à moins de 2 ou 3 vecteurs de Burgers du coeur de la dislocation. En moyenne 1 à 3 deutérons par $\AA$ sont piègés sur les dislocations dans les cristaux déformés. Les mesures indiquent que la géométrie de corrélation rectiligne en baguette s'étend sur 50 à $100 \AA$ en moyenne le long des dislocations. La diffusion observie à partir d'un échantillon de palladium polycristallin bien recuit montre un comportement attendu à partir des surfaces. Cette diffusion est attribuée au deutérium piégé sur les joints de grains dans l'échantillon polycristallin. On calcule une valeur de $0,4 \pm 0,2$ deutérons piégés par $\AA^{2}$ de surface de joint de grains à partir de mesures de diffusion sur un polycristal bien recuit.

Zumammenfasoung - Verformte Palladium-Ein- und Vielkristalle, mit oder ohne gelöstem Deuterium in der Lösungsphase bei Raumtemperatur, werden mittels Neutronenkleinwinkelstreuung untersucht. Der Streuquerschnitt derselben Probe ohne und mit Deuteriumbeladung zeigt ein Verhalten, wie es aus der Deuteriumkorrelation mit Versetzungen, welches zu stabartigen Streustrukturen führt, erwartet wird. Aus diesen Streumessungen folgern wir, da B das eingefangene Deuterium innerhalb von 2 bis 3 Burgersvektoren um den Versetzungskern herum liegt. Im Mittel sind 1 bis 3 Deuteriumatome pro $\AA$ an den Versetzungen in der verformten Probe eingefangen. Die Messungen deuten an, da $B$ die gerade, stabartige Korrelationsgeometrie sich im Mittel über 50 bis $100 \mathrm{~A}$ entlang der Versetzung ausdehnt. Streuung von einer gut ausgeheilten polykristallinen Probe zeigt ein Verhalten, wie es von Oberflächen erwartet wird. Diese Streuung wird dem Deuterium zugeschrieben, welches an Korngrenzen eingefangen ist. Aus den Messungen ergibt sich, daß $0,4 \pm 0,2$ Deuteriumatome pro $\AA^{2}$ vorliegen.

\section{INTRODUCTION}

This paper presents a series of small angle neutron scattering (SANS) measurements from deuteriumloaded single crystal and polycrystalline palladium. Small angle neutron spectroscopy, which is sensitive to non-random deuterium spatial distributions, pro-

†Present address: University of Missouri, Research Reactor Facility, Columbia, MO 65211, U.S.A. vides a direct measurement of deuterium trapped at defects in metals. The effect of trapped deuterium is best represented by a net neutron scattering cross section. In the following we define the net scattering cross section as the scattering difference from the same sample with and without deuterium loading. Net small angle neutron scattering from deuterium trapped at dislocations and at grain boundaries in palladium have been observed and are reported here. 
SANS measurements of deuterium trapped at dislocations in deformed palladium and vanadium have been reported by Kirchheim et al. [1] and by Carstanjen et al. [2]. The only data shown in either reference, a net scattering measurement of vanadium, is presented in Guinier form [3]. Although no data is shown, a distance scale of $39 \AA$ for deuteriumdislocation correlation in palladium is quoted in Ref. [2].

SANS measurements by Heuser et al. [4] from polycrystalline palladium deformed by rolling and a hydride-dehydride cycle exhibit a net scattering signal attributed to deuterium trapped close to long dislocation cores. The deformed samples in reference 4 were examined at room temperature and had deuterium dissolved in the solution phase ( $\alpha$ phase). These data obey a $1 / Q$ scattering law ( $Q=4 \pi / \lambda \sin \theta / 2$ and $\theta$ is the scattering angle) indicative of long, rod-like scattering structures. Ideally, a $1 / Q$ behavior is expected in the limiting case of straight, infinitely long, zero-radius scattering structures. The SANS data in reference 4 were sufficient only to place an upper bound of $30-40 \AA$ on the radial dimension of the rod-like deuterium correlated with dislocations. Volume average dislocation densities in the deformed palladium samples are calculated from a simple relation for the net cross section given in Ref. [4]. The calculated dislocation densities are very reasonable, with an accuracy approaching $10 \%$ in the best case. The key results from reference 4 are reproduced in the Results and Discussion section below.

The measurements presented here for deformed palladium are a significant improvement over previous SANS measurements reported in Ref. [4]. This improvement is primarily the result of better counting statistics obtained in these measurements. These more sensitive measurements showed previously unobserved deviations from a $1 / Q$ behavior. These deviations can be directly related to two separate effects: at low $Q$ the deviations are caused by the finite length of the straight, rod-like deuterium correlation with dislocations while at higher $Q$ the deviations are caused by the non-zero radial width of the distributions. The important parameters describing deuterium correlated with dislocations are determined from a comparison of measured net neutron scattering cross sections to calculated cross sections. A model of the net scattering cross section is presented that explicitly accounts for the radial dimension of the deuterium correlation (defined from the center of the dislocation line outward), length of the straight, rod-like deuterium correlation along the dislocations, and the volume average dislocation density.

Grain boundary trapping of deuterium was investigated in a well annealed polycrystalline palladium sample. A form for the net neutron scattering cross section describing deuterium correlated with grain boundaries is presented. The number of trapped deuterons per unit area of grain boundary is cal- culated for the well annealed polycrystalline sample. Deuterium trapping at grain boundaries in a deformed polycrystalline sample is also analyzed.

In the next section sample preparation, deuterium gas loading techniques, and neutron spectrometer configurations are outlined. Models of the net neutron scattering cross sections from deuterium correlated with dislocations and grain boundaries are then presented. The measured net scattering cross sections are compared to the calculated cross sections in the Results and Discussion section. The focus will be on the parameters describing the deuterium trapping at dislocations and grain boundaries in the palladium samples. Finally, evidence of dislocation scattering in deformed single crystal palladium samples is presented.

\section{EXPERIMENTAL}

Three deformed palladium samples, two single crystal and one polycrystalline, have been prepared. As-grown single crystal samples were taken from a $13 \mathrm{~mm}$ dia. by $75 \mathrm{~mm}$ long rod $(99.999 \%$ purity) supplied by Materials By Metron. These samples had [110] surface normals and were deformed by two methods: cross rolled at $200 \mathrm{~K}$ to $80 \%$ reduction in thickness or given a $100 \%$ hydride-dehydride cycle in a vacuum manifold. These two deformed single crystal samples are respectively referred to as $\mathrm{SC} \mathrm{cw}$ and $\mathrm{SC} \alpha / \beta / \alpha$. The polycrystalline sample was taken from a $6.35 \mathrm{~mm}$ diameter by $25 \mathrm{~mm}$ long rod $(99.96 \%$ purity) supplied by Johnson Matthey Aesar. The as-grown polycrystalline material was rolled to a $70 \%$ reduction in thickness and then annealed in flowing argon at $1000^{\circ} \mathrm{C}$ for $8 \mathrm{~h}$. This procedure produced a well-annealed sample with $150-250 \mu \mathrm{m}$ grain size. The sample was then given a $82 \%$ hydride-dehydride cycle $(82 \%$ of bulk converted to the hydride phase and back again). This sample is referred to as POLY $\alpha / \beta / \alpha$. A well-annealed, undeformed polycrystalline palladium reference sample was also prepared and is referred to as POLY ref. To prepare this sample as-grown polycrystalline rod $(99.95 \%$ purity supplied by Materials By Metron) was rolled to $70 \%$ reduction in thickness and annealed in flowing argon at $1000^{\circ} \mathrm{C}$ for $10 \mathrm{~h}$. A $55-95 \mu \mathrm{m}$ grain size resulted from this procedure.

All samples were cut into three $1 \mathrm{~mm}$ thick by $11 \mathrm{~mm}$ diameter wafers to facilitate deuterium loading by the gas equilibrium technique. The samples were then given a standard mechanical polishing treatment with $1 \mu \mathrm{m}$ alumina used in the last polishing step. All samples were preannealed in a vacuum of $10^{-6}$ torr at $180^{\circ} \mathrm{C}$ for $1.5 \mathrm{~h}$ prior to deuterium loading. Detailed deuterium loading procedures are given in Ref. [4] and will not be covered here. An environmental gas cell with $0.32 \mathrm{~cm}$ thick single crystal silicon windows was used to maintain equilibrium deuterium concentrations in a given sample during the SANS measurements. Once equi- 
librium deuterium concentrations had been achieved in a sample it was quickly transferred to the gas cell. The gas cell was then evacuated and backfilled and with the equilibrium deuterium gas pressure and one atmosphere of nitrogen. After completion of the deuterium-loaded SANS measurement the three wafer sample was heated in vacuum to $120^{\circ} \mathrm{C}$ and pumped free of deuterium for $1-2 \mathrm{~h}$. The sample was then reinserted into the gas cell for an unloaded SANS measurement. Additional measurements were made to obtain sample transmission, empty gas cell background, dark current background, and an absolute intensity calibration from $1 \mathrm{~mm}$ of water.

The SANS measurements reported here were performed on the palladium samples with deuterium dissolved in the $x$ phase. Deuterium solubility enhancement ratios (defined as the ratio of overall deuterium concentration in a defomed sample versus that in an undeformed sample) were 1.37, 1.27, 1.34, and 1.01 for SC $\alpha / \beta / \alpha$, SC cw, POLY $\alpha / \beta / \alpha$, and POLY ref, respectively. The overall deuterium concentrations during the SANS measurement were held at 7550, 6900, 7500 and $5600 \mathrm{ppm}$, respectively. These enhancement ratios are consistent with other solubility measurements of deformed palladium [4-8]. The enhanced deuterium solubility is attributed to deuterium trapped about defects in the deformed samples. Only the enhanced fraction of deuterium about dislocations or grain boundaries will contribute to the net coherent neutron scattering signal at small angles.

The SANS measurements were performed at the Laboratoire Leon Brillouin, CEN-Saclay, France using the small angle spectrometer PACE. The scattering measurements were taken at a neutron wavelength of $6.56 \AA$. The resolution was $10 \% \Delta \dot{\lambda} / \lambda$, where $\Delta \lambda$ is the FWHM of the neutron wavelength distribution. The primary instrumental configuration used had the scattered flight path from the sample to the detector set at $2.5 \mathrm{~m}$. Incident collimation consisted of two cad. mium apertures, $7 \mathrm{~mm}$ and $10 \mathrm{~mm}$ dia., spaced $2.5 \mathrm{~m}$ apart for this configuration with the $7 \mathrm{~mm}$ aperture closest to the sample stage. This instrumental configuration will be referred to as the high $Q$ configuration. In addition, some measurements were performed at $6.56 \AA$ with the scattered flight path extended to $4.69 \mathrm{~m}$ and the incident collimation apertures spaced $5 \mathrm{~m}$ apart. This low $Q$ configuration extended the measured $Q$ range down below $0.01 \AA^{-1}$. The detector consisted of 30 concentric rings of $1 \mathrm{~cm}$ width with the first ring having a centerline diameter of $6 \mathrm{~cm}$. The samples were centered between two $9 \mathrm{~mm}$ inner dia. cadmium apertures inside the gas cell. All SANS measurements were performed at room temperature and were $4.6 \mathrm{~h}$ in duration.

\section{THEORETICAL FRAMEWORK}

A form for the differential macroscopic scattering cross section per steradian describing non-random spatial distributions of deuterium about dislocations and grain boundaries in palladium is calculated in this section. Coherent small angle neutron scattering results from non-random density fluctuations of the target nuclei extending over distances upwards from a few times the neutron wavelength. The differential macroscopic scattering cross section is proportional to the square of the Fourier transform of these density fluctuations.

\section{Deuterium-deuterium correlation at dislocations}

The total differential macroscopic scattering cross section of a deformed, deuterium-loaded palladium sample consists of four components; defect scattering, defect-deuterium correlation scattering, deuterium-deuterium correlation scattering, and incoherent scattering from palladium and deuterium. By defect we mean dislocations in the single crystal samples and dislocations or grain boundaries in the polycrystalline samples. SANS measurements of SC $\alpha / \beta / \alpha, \mathrm{SC} \mathrm{cw}$, and a single crystal reference sample, all without deuterium loading, are presented in the next section. These measurements show that dislocation scattering alone is expected only at the lowest $Q$ limit of the high $Q$ configuration. It follows that over almost the entire $Q$ range of the deuterium loaded SC $\alpha / \beta / \alpha$ and $\mathrm{SC} \mathrm{cw}$ measurements, dislocation scattering and dislocation-deuterium correlation scattering will not contribute to the total differential cross section. In addition, the definition of a net macroscopic scattering cross section eliminates the palladium incoherent scattering contribution and all other scattering events not related to the presence of deuterium. The incoherent scattering from deuterium within the palladium samples is infinitesimally small. The net macroscopic scattering cross section for the SC $\alpha / \beta / \alpha$ and $\mathrm{SC} \mathrm{cw}$ samples is written as

$$
\mathrm{d} \Sigma / \mathrm{d} \Omega(\mathbf{Q})=N_{\mathrm{d}} b^{2}\left\langle\left|\int_{V} \mathrm{~d}^{3} \mathbf{r} C(\mathbf{r}) \mathrm{e}^{\mathbf{Q} \cdot r}\right|^{2}\right\rangle .
$$

In equation (l) $\mathbf{Q}=\mathbf{k}^{\prime}-\mathbf{k}$, where $\mathbf{k}$ and $\mathbf{k}^{\prime}$ are the incident and scattered neutron wave vectors, respectively, $\mathbf{r}$ is the deuterium atom position vector, $C(\mathbf{r})$ is the distribution of trapped deuterons at a dislocation per unit volume, $b$ is the bound atom coherent scattering length of a deuteron, $N_{\mathrm{d}}$ is the number of dislocations per unit volume, and $V$ is the volume associated with a single dislocation.

The SANS measurement of the deuterium-loaded POLY $\alpha / \beta / \alpha$ sample is complicated by scattering from deuterium correlated with grain boundaries that occurs below $Q \sim 0.025 \AA$ '. Therefore, equation I describes the net macroscopic scattering cross section of the POLY $\alpha / \beta / \alpha$ sample, but only above $Q \sim 0.025 \AA^{-1}$. (Scattering from deuterium correlation with grain boundaries has been measured separately in the well annealed POLY ref sample and is presented in the next section. Theoretical treatment 
of scattering from deuterium correlation with grain boundaries is given below.)

The integration in equation (1) is performed using the cylindrical coordinates $z, \phi$, and $\mathbf{R}$. These coordinates are defined with the $z$-axis along the dislocation line, $\mathbf{R}$ as the projection of $\mathbf{r}$ onto the $x-y$ plane, and $\phi$ as the angle between $R$ and the $x$-axis. $C(\mathbf{r})$ is defined as a function of $R$ such that $C\left(R \leqslant R_{0}\right)=C$ and $C\left(R>R_{0}\right)=0$. The net macroscopic scattering cross section is now given by

$$
\begin{aligned}
\mathrm{d} \Sigma / \mathrm{d} \Omega(Q)= & \pi^{2} b^{2} N_{\mathrm{d}} C^{2} R_{0}^{4} \exp \left(\frac{-Q^{2} R_{0}^{2}}{4}\right) \\
& \times \int_{0}^{\infty} \mathrm{d} L p(L) \int_{-1}^{1} \mathrm{~d} \mu \frac{\sin ^{2} \frac{Q L \mu}{2}}{(Q \mu)^{2}} .
\end{aligned}
$$

In equation (2), $\mu$ is the cosine of the angle between $\mathbf{Q}$ and the $z$-axis, and $p(L)$ is the distribution of deuterium correlation lengths along the dislocation. The parameter $L$ is a measure of distance over which the rod-like deuterium correlation remains straight. (Deviations will result from dislocation curvature for example.) The $p(L)$ distribution accounts for this deviation from straight line behavior. The calculation of the net scattering cross section is rather insensitive to the functional form of $p(L)$ for the $Q$ range used in this experiment. For simplicity we use an exponential distribution, $p(L)=\left(1 / L_{0}\right) \exp \left(-L / L_{0}\right)$ where $L_{0}$ is a constant. The net macroscopic scattering cross section is given in final form as

$$
\mathrm{d} \Sigma / \mathrm{d} \Omega(Q)=\frac{2 b^{2} \rho_{\mathrm{D}}^{2}}{Q \rho_{\mathrm{d}}} \exp \left(\frac{-Q^{2} R_{0}^{2}}{4}\right) \tan ^{-1} Q L_{0} .
$$

In equation (3), $\rho_{\mathrm{D}}$ is the density for the total enhanced fraction of deuterium dissolved in the sample given by $\rho_{\mathrm{D}}=\pi R_{0}^{2} L_{0} N_{\mathrm{d}} C$ and $\rho_{\mathrm{d}}$ is the volume average dislocation density of the sample in $\mathrm{cm} / \mathrm{cm}^{3}$ given by $\rho_{\mathrm{d}}=L_{0} N_{\mathrm{d}}$.

Equation (3) gives the net differential neutron scattering cross section for a straight, rod-like deuterium distribution about dislocations. Equation (3) simplifies to $\mathrm{d} \Sigma / \mathrm{d} \Omega \propto 1 / Q$ in the limit as $R_{0}$ goes to zero and $L_{0}$ goes to infinity. A pure $1 / Q$ form then is the result expected for zero-radius deuterium distributions about infinitely long, straight dislocation lines. The exponent and inverse tangent factors can, therefore, be viewed as corrections to a pure $1 / Q$ form.

\section{Deuterium-deuterium correlation at grain boundaries}

The net macroscopic differential neutron scattering cross section from deuterium trapped at grain boundaries is written as

$$
\mathrm{d} \Sigma / \mathrm{d} \Omega(\mathbf{Q})=\frac{1}{V}\left\langle\left|b s \int_{V} \mathrm{~d}^{3} \mathrm{r} \mathrm{e}^{\mathrm{Q} \cdot \mathrm{r}} \delta\left(\mathbf{r}-\mathbf{R}_{\mathrm{g}}\right)\right|^{2}\right\rangle
$$

where $s$ is defined as the number of trapped deuterons per unit area of grain boundary, $R_{\mathrm{B}}$ is the average grain radius, and $V$ is the volume of a grain of radius
$R_{\mathrm{g}}$. Equation (4) is the scattering cross section from a spherical shell of trapped deuterons for which the radius of curvature is large and the width of the shell is small compared to $1 / Q$. The net macroscopic scattering cross section for trapped deuterium at grain boundaries is

$$
\mathrm{d} \Sigma / \mathrm{d} \Omega(Q)=\frac{6 \pi b^{2} s^{2}}{R_{\mathrm{g}} Q^{2}} .
$$

\section{RESULTS AND DISCUSSION}

\section{Deuterium correlated with dislocations}

The net macroscopic neutron scattering cross sections for $\mathrm{SC} \alpha / \beta / \alpha$ and $\mathrm{SC} \mathrm{cw}$ are shown in Figs 1 and 2 , respectively. Figure 3 shows the net macroscopic neutron scattering cross section for POLY $\alpha / \beta / \alpha$. The measured net scattering cross sections shown in Figs 1, 2, and 3 are all in absolute units and were taken in the high $Q$ configuration. The solid curves in Figs 1, 2 and 3 are plots of equation (3) with the magnitude of $\rho_{\mathrm{d}}$ determined by a best fit to the data. The calculated cross section fits the measured scattering data very well, except for the lower $Q$ portion of Fig. 3. Here additional scattering from deuterium correlation with grain boundaries is present. The dotted line in Figs 1 and 2 represents $\mathrm{d} \Sigma / \mathrm{d} \Omega \propto 1 / Q$, normalized above $Q=0.05 \AA^{-1}$. Differences between equation (3) and $1 / Q$ above $0.05 \AA^{-1}$ seem slight in Figs 1 and 2. An exploded view of this $Q$ region would show noticeable differences, however. The radial dimension $\left(R_{0}\right)$, the average correlation length over which the deuterium correlation with dislocations remains straight $\left(L_{0}\right)$, and the volume average dislocation density $\left(\rho_{\mathrm{d}}\right)$ determined from the fit of equation (3) are shown in Table 1.

The deuterium density, $\rho_{\mathrm{D}}$, is the defect-enhanced fraction, i.e. the difference between the total density in a deformed sample and the density in an undeformed sample. The total deuterium density was calculated from the total pressure change during gas loading. Sievert's law was used to calculate the bulk deuterium density from the gas pressure reached at equilibrium. (The solubility constants used for deuterium dissolved in palladium have been confirmed by repeated gas equilibrium measurements.) Errors in $\rho_{\mathrm{D}}$ reflect errors in the gas loading measurements. The exponent and inverse tangent factors in equation (3) predominate in two different $Q$ ranges. Data below $Q \sim 0.05 \AA^{-1}$ are influenced mostly by the finite length of the straight, rod-like deuterium correlations. Above $Q \sim 0.05 \AA^{-1}$, the non-zero radial dimension of the deuterium distribution about dislocations is the important correction. A single parameter $\chi^{2}$ fitting procedure was used separately below and above $Q=0.05 \AA^{-1}$ to find the optimum values of $L_{0}$ and $R_{0}$, respectively. Errors in $L_{0}$ and $R_{0}$ were estimated from the shape of the $\chi^{2}$ vs $L_{0}$ or $R_{0}$ curves. Once $L_{0}$ and $R_{0}$ were known, the $\rho_{\mathrm{d}}$ values and associated uncertainties were 


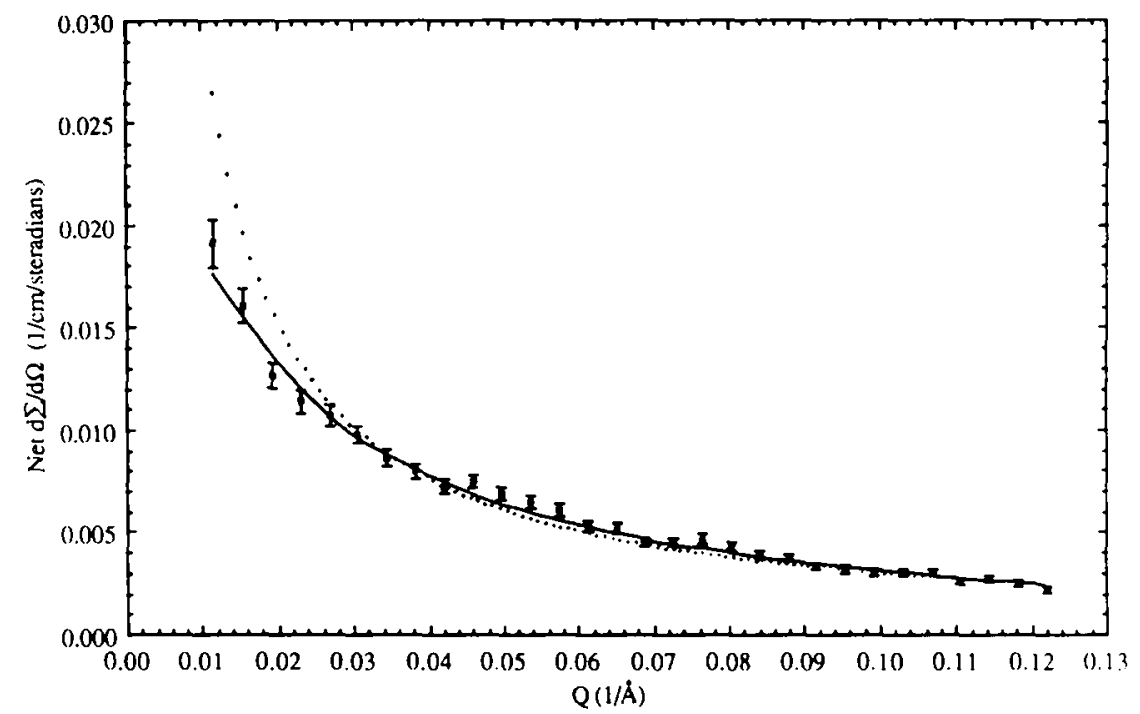

Fig. 1. Net $\mathrm{d} \Sigma / \mathrm{d} \Omega$ vs $Q$ for $\mathrm{SC} \alpha / \beta / \alpha$; solid square symbols with error bars are measured data (high $Q$ configuration), solid curve is equation (3) with $R_{0}=7 \pm 2 \AA$ and $L_{0}=100 \pm 20 \AA$, dotted curve is $\mathrm{d} \Sigma / \mathrm{d} \Omega \propto 1 / Q$.

calculated from the best-fit slope of $\mathrm{d} \Sigma / \mathrm{d} \Omega$ vs $(1 / Q)$ $\exp \left[\left(-Q^{2} R_{0}^{2}\right) / 4\right] \tan ^{-1}\left(Q L_{0}\right)$. Another method of determining $R_{0}$ is demonstrated in Fig. 4 , which shows a plot of $\ln [\mathrm{d} \Sigma / \mathrm{d} \Omega \times Q]$ vs $Q^{2}$ for the $\mathrm{SC} \alpha / \beta / \alpha$ sample. Based on equation (3), above the $Q$ range where the length correction is significant, $R_{0}$ can be calculated directly from the slope of the data shown in Fig. 4. This method gives results for all three net scattering measurements that are consistent with the $R_{0}$ values determined from the $\chi^{2}$ routine.

The dislocation densities shown in Table 1 are realistic for a heavily deformed metal and are also consistent with other SANS measurements of deformed palladium, as discussed below. Notice the uncertainty of the dislocation densities shown in
Table 1 are low, approaching $5 \%$ in the best case. Preliminary transmission electron microscopy (TEM) analysis indicate volume averaged dislocation densities for cold rolled and hydride-dehydride cycled single crystal palladium of roughly $5 \times 10^{11} 1 / \mathrm{cm}^{2}$. TEM analysis also shows dramatic differences in dislocation arrangements between the two methods of deformation. As expected for a high stacking fault metal, the severe cross rolling results in a high degree of dislocation cellularization. On the other hand, the dislocation substructure in the cycled material is much more uniform.

Based on TEM analysis alone it is not possible to determine if the absolute value of $L_{0}, 75$ and $100 \AA$ in the cycled samples and $50 \AA$ in the cross rolled

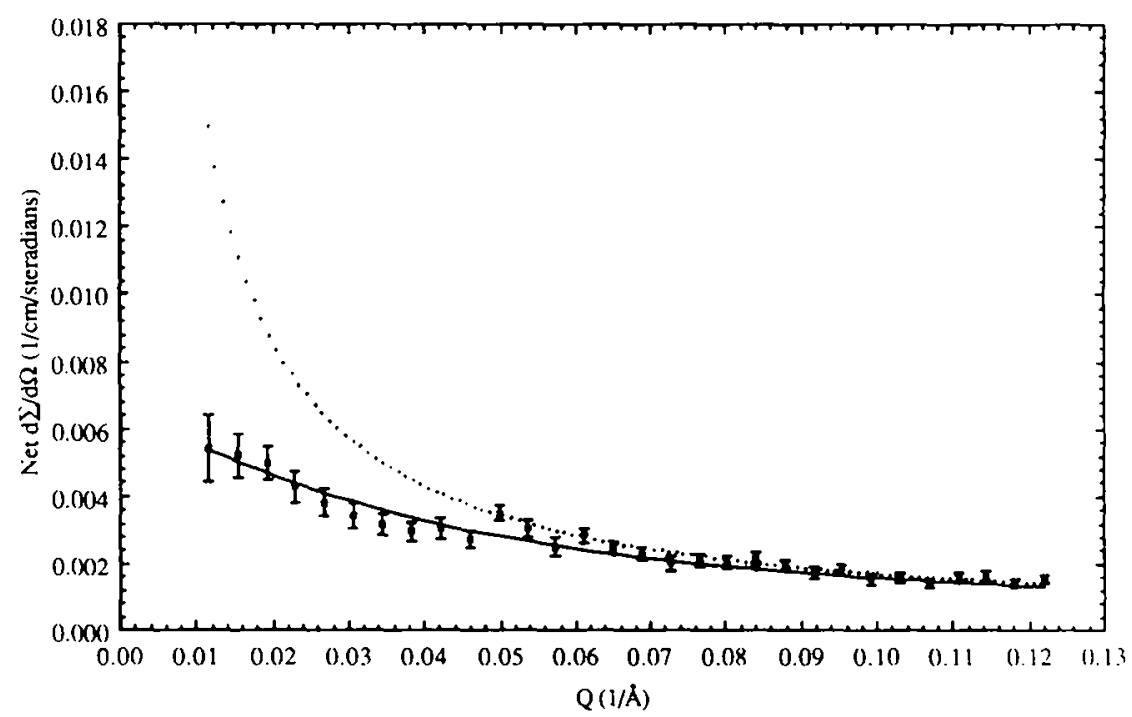

Fig. 2. Net $\mathrm{d} \Sigma / \mathrm{d} \Omega$ vs $Q$ for $\mathrm{SC} \mathrm{cw}$; solid square symbols with error bars are measured data (high $Q$ configuration), solid curve is equation (3) with $R_{0}=4 \pm 2 \AA$ and $L_{0}=50 \pm 10 \AA$, dotted curve is $\mathrm{d} \Sigma / \mathrm{d} \Omega \propto 1 / Q$. 


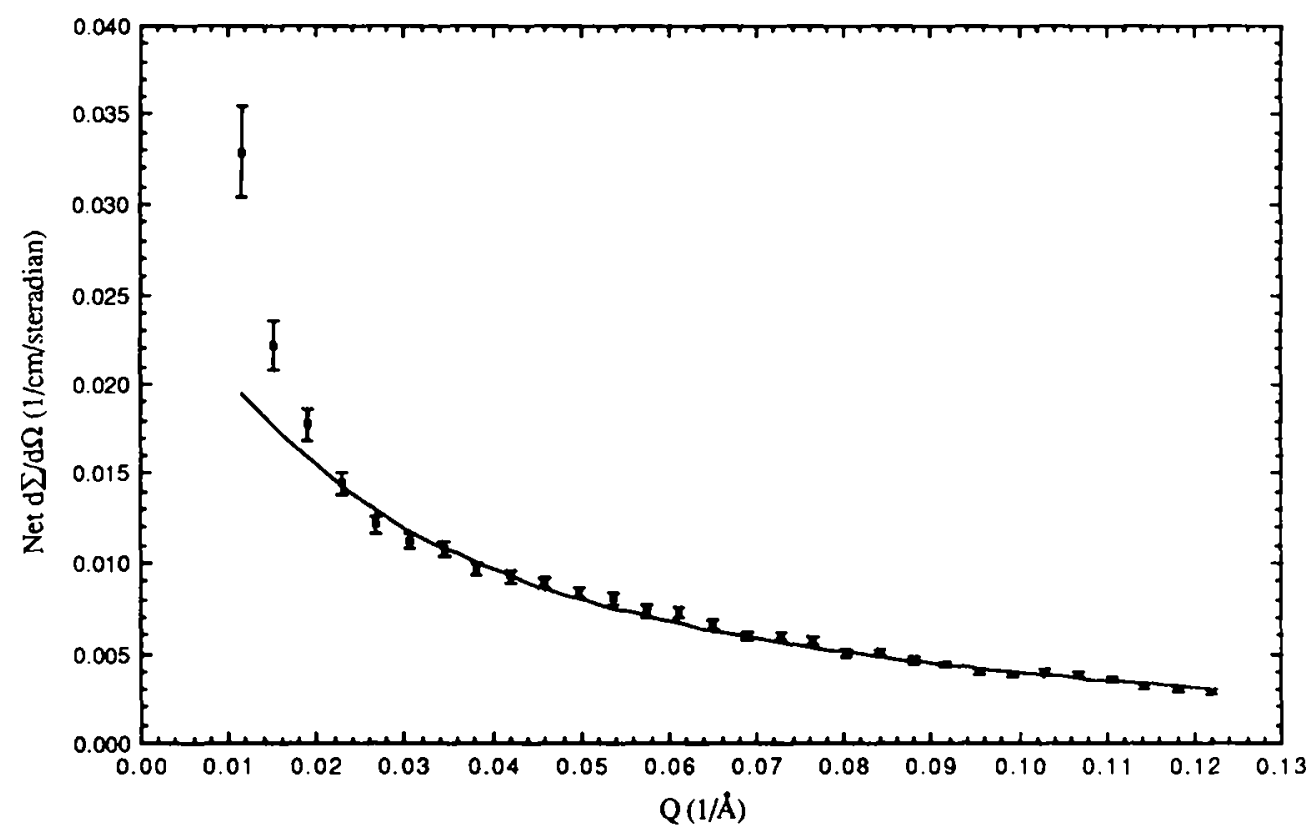

Fig. 3. Net $\mathrm{d} \Sigma / \mathrm{d} \Omega$ vs $Q$ for POLY $\alpha / \beta / \alpha$; solid square symbols with error bars are measured data (high $Q$ configuration), solid curve is equation (3) with $R_{0}=8 \pm 2 \AA$ and $L_{0}=75 \pm 15 \AA$.

Table 1. Experimental parameters describing deuterium correlation with dislocations in palladium

\begin{tabular}{lccccc}
\hline $\begin{array}{l}\text { Sample } \\
\text { ID }\end{array}$ & $\begin{array}{c}\rho_{\mathrm{D}} \\
\left(\times 10^{20} \mathrm{l} / \mathrm{cm}^{3}\right)\end{array}$ & $\begin{array}{c}\rho_{\mathrm{d}} \\
\left(\times 10^{11} \mathrm{l} / \mathrm{cm}^{2}\right)\end{array}$ & $\begin{array}{c}R_{0} \\
(\AA)\end{array}$ & $\begin{array}{c}L_{\mathrm{L}} \\
(\AA)\end{array}$ & $\begin{array}{c}\text { Deuterons per } \AA \\
\text { trapped at dislocations }\end{array}$ \\
\hline SC $\alpha / \beta / \alpha$ & $1.37 \pm 0.04$ & $6.9 \pm 0.4$ & $7 \pm 2$ & $100 \pm 20$ & $2.0 \pm 0.1$ \\
SC cw & $0.96 \pm 0.03$ & $7.5 \pm 0.7$ & $4 \pm 2$ & $50 \pm 10$ & $1.3 \pm 0.1$ \\
POLY $\alpha / \beta / \alpha$ & $1.23 \pm 0.12$ & $4.2 \pm 0.8$ & $8 \pm 2$ & $75 \pm 15$ & $2.9 \pm 0.6$ \\
\hline
\end{tabular}

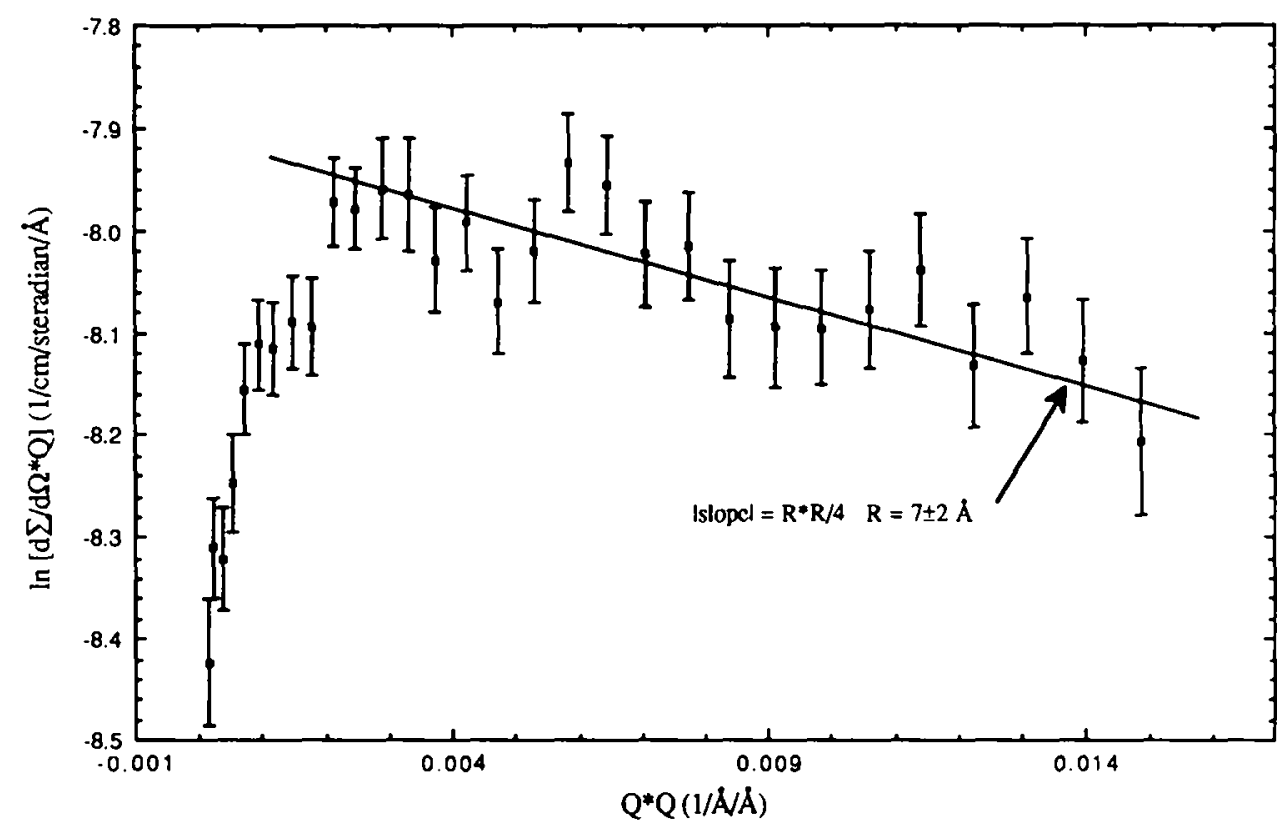

Fig. 4. $\ln \left[\frac{\mathrm{d} \Sigma}{\mathrm{d} \Omega} \times Q\right]$ vs $\ln Q * Q$

for $\mathrm{SC} \alpha / \beta / \alpha$; solid square symbols with error bars are measured data (high $Q$ configuration). Absolute value of slope above $Q \sim 0.04 \AA^{-1}$ is equal to $R^{2} / 4$. 
Table 2. SANS results for polycrystalline samples from Ref. [4].

\begin{tabular}{lccc} 
Sample & $\begin{array}{c}\rho_{\mathrm{D}} \\
\text { ID }\end{array}$ & $\begin{array}{c}\rho_{\mathrm{d}} \\
\left(\times 10^{20} 1 / \mathrm{cm}^{3}\right)\end{array}$ & $\begin{array}{c}\text { Deuterons per } \AA \\
\text { trapped at } \\
\text { dislocations }\end{array}$ \\
\hline$(\alpha / \beta / \alpha) !$ & $1.86 \pm 0.06$ & $6.4 \pm 0.7$ & $2.9 \pm 0.3$ \\
$(\alpha / \beta / \alpha) 2$ & $1.13 \pm 0.03$ & $4.3 \pm 0.9$ & $2.6 \pm 0.6$ \\
$\mathrm{cw} \mathrm{1}$ & $0.93 \pm 0.03$ & $5.2 \pm 1.7$ & $1.8 \pm 0.6$ \\
$\mathrm{cw} \mathrm{2}$ & $1.05 \pm 0.04$ & $12 \pm 6$ & $0.9 \pm 0.5$ \\
\hline
\end{tabular}

sample, are appropriate. Furthermore, the possible mechanisms responsible for the finite correlation lengths, dislocation curvature and dislocation jogs for example, are very complicated. The systematic difference in $L_{0}$ values evident in Table 1 , especially for the single crystal samples, may be real. This difference in correlation length could be related to the differences in dislocation cell structure discussed above. It seems plausible that the entanglement of dislocations found in cell walls could limit the straight, rod-like deuterium correlations measured in the cross rolled sample, SC cw.

The important results from Ref. [4] are reproduced in Table 2. These SANS measurements were performed with two sets of polycrystalline palladium samples deformed by cross rolling (samples $\mathrm{cw} 1$ and cw 2) and by a hydride-dehydride cycle (samples $(\alpha / \beta / \alpha) 1$ and $(\alpha / \beta / \alpha) 2)$. The scattering measurements shown were made on the small angle diffractometer (SAD) at the Argonne National Laboratory time of flight source, IPNS. Even though the point to point statistics are only fair for the SAD measurements, it is possible to calculate accurate volume average dislocation densities from a best-fit slope of $\mathrm{d} \Sigma / \mathrm{d} \Omega$ vs $1 / Q$.

As described in Ref. [4], $100 \%$ of $(\alpha / \beta / \alpha) 1$ and $67 \%$ of $(x / \beta / x) 2$ were cycled through the solid solution-hydride two phase region. The dislocations in the $\alpha / \beta / \alpha$ samples are created as the solid solution- hydride phase boundary propagates through the material. We then expect the dislocation density of $(\alpha / \beta / \alpha) 2$ to be $66 \%$ of $(\alpha / \beta / \alpha) 1$. Table 2 shows this is the case. Another comparison can be made between POLY $\alpha / \beta / \alpha$ measured on PACE and $(\alpha / \beta / \alpha) 1$ measured on SAD. In the preparation of POLY $\alpha / \beta / \alpha$ approximately $82 \%$ of the bulk was cycled through the two phase region. Table 1 shows the dislocation density of POLY $\alpha / \beta / \alpha$ is $66 \%$ of $(\alpha / \beta / \alpha) 1$. The difference in this case is within experimental error.

Small discrepancies exist between the dislocation densities shown in Table 2 and the dislocation densities given in Ref. [4]. These discrepancies are due to a reevaluation of the SAD data in a manner consistent with calculations shown Table 1 and do not affect the discussion in the preceding paragraph.

\section{Deuterium correlated with grain boundaries}

Deuterium trapping at grain boundaries in palladium was investigated by measuring the net scattering response of the POLY ref sample in the low $Q$ configuration. This measured net scattering cross section is shown in Fig. 5 and is, again, in absolute units. The solid curve in Fig. 5 represents equation (5) with $s$ adjusted to fit the experimental data. The measured cross section of POLY ref has a slightly stronger $Q$ dependence than that given by equation (5). An average value of $s=0.4 \pm 0.2$ trapped deuterons per $\AA^{2}$ of grain boundary area is calculated from the measured data below $Q=0.025 \AA^{-1}$. An average grain radius of $38 \mu \mathrm{m}$ was used in this calculation. The total enhanced fraction deuterium density, $\rho_{\mathbf{D}}$ for deuterium trapped at grain boundaries is given by; $\rho_{\mathrm{D}}=3 s / R_{\mathrm{g}}$. For the POLY ref sample, $\rho_{\mathrm{D}}=3.2 \times 10^{18} \mathrm{~cm}^{-3}$. This value of $\rho_{\mathrm{D}}$ represents

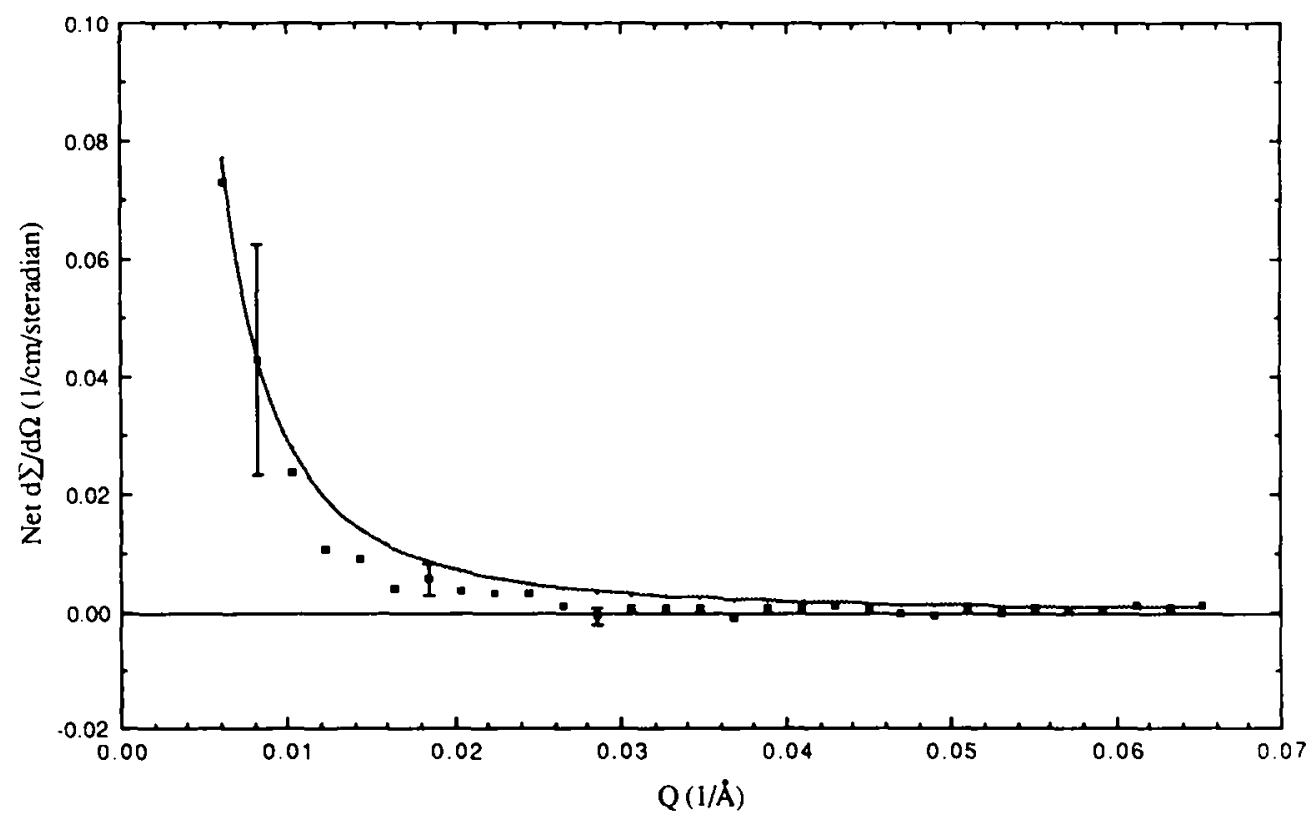

Fig. 5. Net $\mathrm{d} \Sigma / \mathrm{d} \Omega$ vs $Q$ for POLY ref; solid square symbols with error bars are measured data (low $Q$ configuration), solid curve is equation (5) with $s=0.4 \pm 0.2 \AA^{-2}$. 
Table 3. Grain boundary trapping parameters

\begin{tabular}{lcccc}
\hline Sample & $\begin{array}{c}R_{g_{6}} \\
\text { ID }\end{array}$ & $\begin{array}{c}Q \\
\left(\times 10^{-1} \mathrm{~m}\right)\end{array}$ & $\begin{array}{c}\text { Excess } \mathrm{d} \Sigma / \mathrm{d} \Omega \\
\left(\mathrm{Am}^{-1}\right)\end{array}$ & $\begin{array}{c}s \\
\left(\text { deuterons per } A^{2}\right)\end{array}$ \\
\hline POLY ref & 38 & $Q<0.025$ & - & $0.4 \pm 0.2$ \\
POLY $\alpha / \beta / \alpha$ & 100 & 0.0115 & 0.0134 & $0.5 \pm 0.1$ \\
POLY $\alpha / \beta / \alpha$ & 100 & 0.0153 & 0.0045 & $0.4 \pm 0.2$ \\
POLY $x / \beta / \alpha$ & 100 & 0.0192 & 0.0019 & $0.3 \pm 0.2$ \\
\hline
\end{tabular}

approximately $1 \%$ of the bulk deuterium density in POLY ref during the SANS measurement. This is consistent with the solubility enhancement ratio of 1.01 measured for this sample.

As stated earlier, the effect of deuterium trapped at grain boundaries in the POLY $\alpha / \beta / \alpha$ sample was also evident at low $Q$ in Fig. 3. The number of trapped deuterons per unit grain boundary area can be estimated for this sample using equation (5). This calculation and the POLY ref result are shown in Table 3 below. The excess scattering cross section for POLY $\alpha / \beta / \alpha$ shown in Table 3 is the difference between the lowest three measured data points and the solid curve of Fig. 3. An average grain radius of $100 \mu \mathrm{m}$ is used, corresponding to grain size measurements for this sample. Considering the crudeness of the calculation for the POLY $\alpha / \beta / \alpha$ sample, the agreement between the two samples is to be noted.

\section{Evidence of dislocation scattering without deuterium loading}

Figure 6 shows the total macroscopic neutron scattering cross section per steradian for the two deformed single crystal samples. SC $\alpha / \beta / \alpha$ and SC $\mathrm{cw}$, and an undeformed single crystal reference sample, SC ref. The single crystal reference sample was taken from the same starting stock, and had the same orientation as the deformed single crystal samples. The measurements shown in Fig. 6, taken in the high $Q$ configuration, are all without deuterium loading. Although the statistics are marginal an increase in scattering is evident at the lower $Q$ limit for the deformed samples. We attribute this to the presence of dislocations created in the deformation process. In view of the very limited nature of the data, no detailed analysis is yet warranted for these observations.

Figure 7 shows the total scattering cross section for POLY $\alpha / \beta / \alpha$ and POLY ref in ln-ln form measured in the low $Q$ configuration without deuterium loading. At low $Q$ both samples exhibit a very strong scattering behavior proportional to $1 / Q^{4}$, masking out any additional scattering that may be present due to dislocations. According to Atkinson et al. [9] neutron scattering from edge dislocations is expected to show a $1 / Q^{3}$ behavior. The contribution from screw dislocations is expected to be a second order effect. The strong $1 / Q^{4}$ scattering from our polycrystalline samples naturally complicates any attempt to observe dislocation scattering alone. The $1 / Q^{4}$ behavior in Fig. 7 is typical of coherent scattering from isolated particles [3] and is possibly the result of large

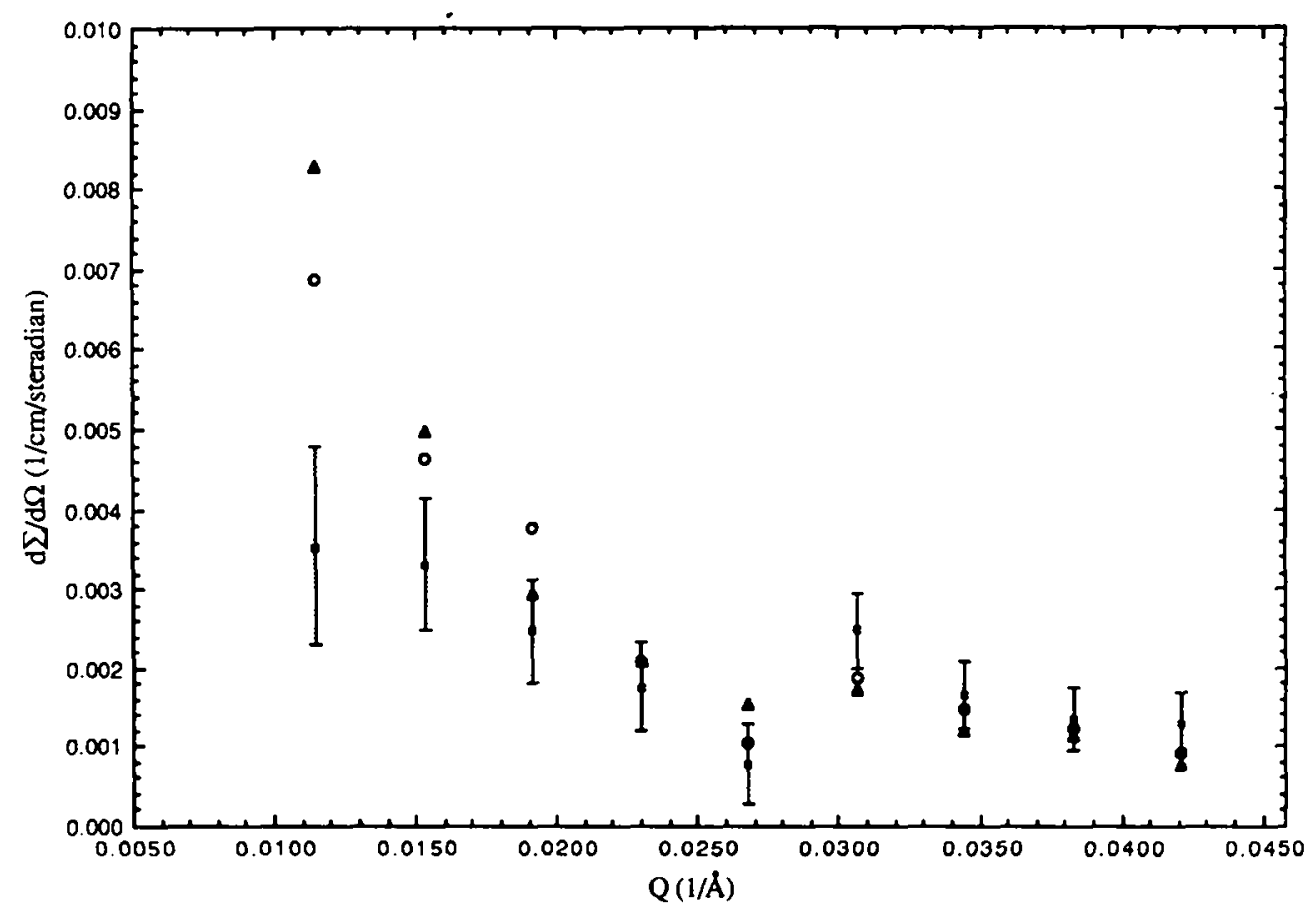

Fig. 6. $\mathrm{d} \Sigma / \mathrm{d} \Omega$ vs $Q$ for $\mathrm{SC} \mathrm{cw}$ (open triangle symbols), $\mathrm{SC} \alpha / \beta / \alpha$ (open circle symbols), and SC ref (solid square symbols). Measurements performed in the high $Q$ configuration. 


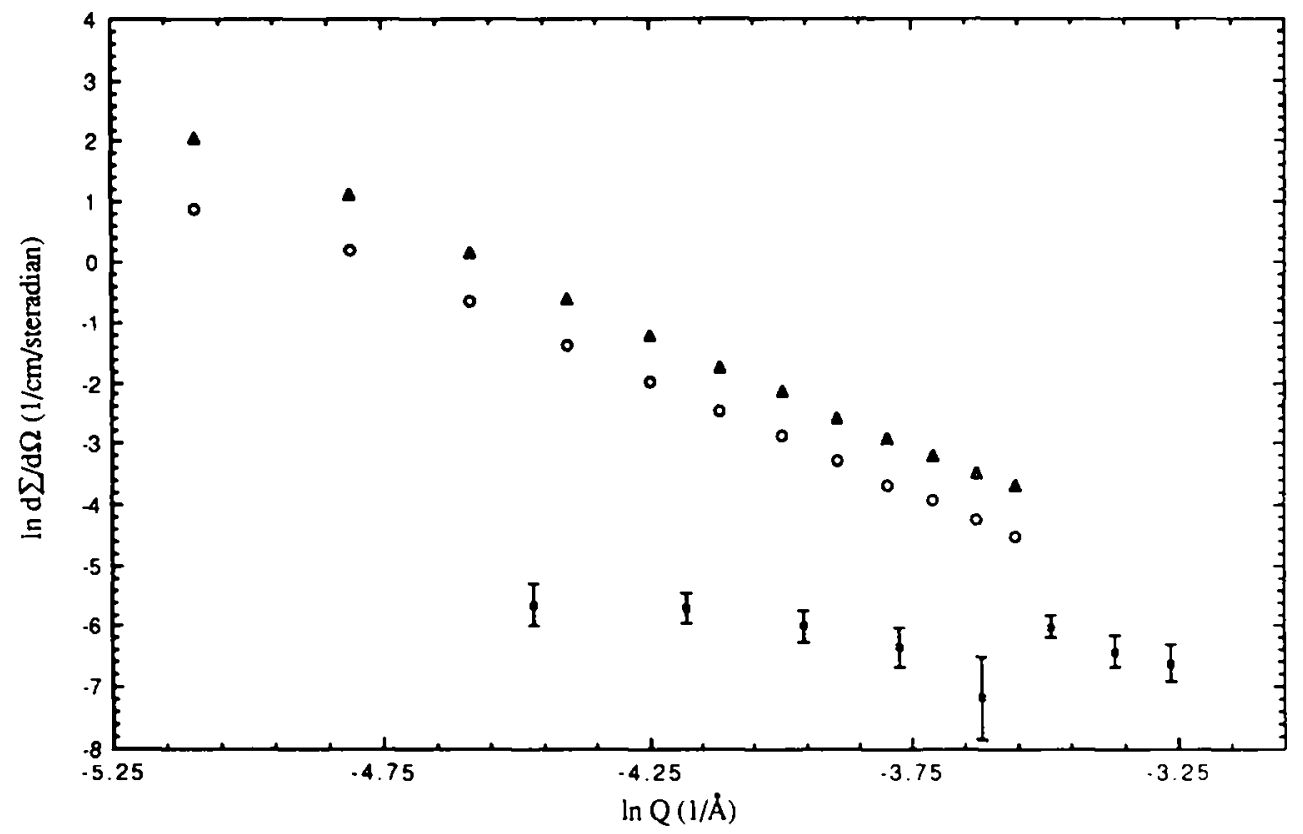

Fig. 7. In $\mathrm{d} \Sigma / \mathrm{d} \Omega$ vs $\ln Q$ for POLY ref (open triangle symbols), POLY $\alpha / \beta / \alpha$ (open circle symbols), and SC ref (solid square symbols). The POLY ref and POLY $\alpha / \beta / \alpha$ samples were measured in the low $Q$ configuration and the SC ref sample was measured in the high $Q$ configuration.

voids or inclusions (by large we mean diameters much greater than $1 / Q$ ) thought to be present in the polycrystalline material. The starting material for the two polycrystalline samples came from different suppliers and undoubtedly had different number densities of $1 / Q^{4}$ scattering objects. This would account for the difference in cross section magnitude between the two polycrystalline samples. The total scattering response from $\mathrm{SC}$ ref, measured in the high $Q$ configuration without deuterium loading, is shown for comparison in Fig. 7. The response of SC ref is very low compared to the polycrystalline samples and does not exhibit a $1 / Q^{4}$ behavior.

\section{CONCLUSIONS}

1. We have modeled deuterium correlation with dislocations in palladium with a rod-like geometry. This geometry is consistent with our understanding of the spatial distribution of trapped deuterium about dislocations. Calculated net differential macroscopic scattering cross sections per steradian match the measured net scattering data very well. The net scattering response from the deformed, deuteriumloaded single crystal and polycrystalline palladium samples is, therefore, attributed to deuterium correlation with dislocations forming rod-like scattering structures.

2. The trapped deuterium appears to be tightly bound to the dislocation core region. The radial extent of the deuterium distribution about dislocations is not greater than 2 or 3 Burgers vectors. In addition, the measurements indicate the straight, rod-like correlation geometry extends on average
50-100 $\AA$ along the dislocations. Systematic differences in the average length of the deuterium correlation with dislocations may be present, revealing the difference in the method of deformation-cross rolling vs. hydride-dehydride cycling.

3. An absolute normalization of the measured net macroscopic scattering cross sections gives accurate volume averaged dislocation densities for each deformed palladium sample. In the case of the $\alpha / \beta / x$ polycrystalline samples (both PACE and SAD measurements) the calculated dislocation densities are approximately proportional to the fraction of the bulk material that underwent the hydride-dehydride cycle. We believe this is strong evidence that the dislocation densities calculated from the measured net scattering cross section are very accurate.

4. A net neutron scattering cross section having a $1 / Q^{2}$ behavior has been measured for a well annealed polycrystalline palladium sample. We believe this is due to deuterium trapped at grain boundaries in the polycrystalline sample. Net scattering in excess of that expected from deuterium correlated at dislocations was also observed at low $Q$ in the deformed polycrystalline measurement. This excess scattering is also attributed to deuterium trapped at grain boundaries. The calculated number of trapped deuterons per unit grain boundary area is approximately $0.4 \AA^{-2}$ in each of these polycrystalline samples.

5. Dislocation scattering alone has been observed at low $Q$ in deformed single crystal palladium samples. Large $1 / Q^{4}$ scattering, possibly due to the presence voids or inclusions, interferes with our ability to observe dislocation scattering in the polycrystalline samples. 
We believe the measurements presented here demonstrate the viability of the SANS technique to directly measure spatial correlations of deuterium trapped at dislocations and grain boundaries on a quantitative scale. It is also our belief the application of this SANS technique to other materials of engineering importance can provide useful information.

Acknowledgements - We wish to acknowledge the support of G. Jannink and J. P. Cotton for making the Saclay measurements possible. For the ANL data work was supported by the U.S. Department of Energy, BES-Materials Science under contract W-31-109-ENG-38.

\section{REFERENCES}

1. R. Kirchheim, X. Y. Huang, H.-D. Carstanjen and J. J. Rush, Chemistry and Physics of Fracture, NATO ASI Series, Series E, No. 130, FRG, p. 580 (1987).
2. H.-D. Carstanjen, X. Y. Huang, W. Kieninger, R. Kirchheim, J. Rush, T. Udovic, J. Glinka and A. Petry, Z. Physik Chem. Neue Folge 163, 203 (1989).

3. A. Guinier and G. Fournet, Small Angle Scattering of $X$-rays. Wiley, New York (1955).

4. B. J. Heuser, G. C. Summerfield, J. S. King and J. E. Epperson, Neutron Scattering for Materials Science, Mater. Res. Soc. Proc., Vol. 166, p. 273 (1989).

5. T. B. Flanagan, J. F. Lynch, J. D. Clewley and B. von Turkovich, Scripta metall. 9, 1063 (1975).

6. T. B. Flanagan and S. Kishimoto, Electronic Structure and Properties of Hydrogen in Metals (edited by P. Jena and L. B. Satterthwaite), NATO Conf. Series. VI, Materials Science, Richmond, Va, Vol. 6, p. 623 (1982).

7. J. F. Lynch, J. D. Clewley, T. Curran and T. B. Flanagan, J. less-common Metals. 55, 153 (1977)

8. R. Kirchheim, Acta metall. 29, 835 (1981); 29, 845 (1981).

9. H. H. Atkinson and P. B. Hirsch, Phil. Mag. 3, 213 (1958). 